\title{
The Micromegas chambers for the ATLAS New Small Wheel upgrade
}

\author{
Deb Sankar Bhattacharya, on behalf of the ATLAS Muon \\ Collaboration $\ddagger$ \\ Julius-Maximilians-University of Würzburg, Germany \\ E-mail: deb.sankar.bhattacharya@cern.ch
}

February 2020

\begin{abstract}
The ATLAS collaboration at the LHC has chosen the resistive Micromegas (MM) technology, along with the small-strip Thin Gap Chambers (sTGC), for the high luminosity upgrade of the first muon station in the high-rapidity region, the so called New Small Wheel (NSW) project. After the R\&D, design and prototyping phase, the first series production Micromegas quadruplets are being constructed at the involved construction sites in France, Germany, Italy, Russia and Greece. At CERN, the final validation and the integration of the modules in Sectors are in progress. These are big steps forward for the installation of the NSW foreseen for the LHC long shutdown in 2019 and 2020. The construction of the four types of large size quadruplets, all having trapezoidal shapes with surface areas between 2 and $3 \mathrm{~m}^{2}$, will be reviewed. Achieving the requirements for these detectors was revealed to be even more challenging than expected, when scaling from the small prototypes to large dimensions. We will describe the encountered problems, to a large extent common to other micro-pattern gaseous detectors, and the adopted solutions. Final quality assessment of the High-Voltage stability of the modules, with and without irradiation, will be presented together with the most relevant steps and results.
\end{abstract}

Keywords: ATLAS, New Small Wheel, Muon, Tracking, Triggering, Micromegas

\section{Introduction}

After successfully finishing Run 2 operation at the end of 2018, the Large Hadron Collider (LHC) [1] complex is now preparing for Run 3. The instantaneous beam luminosity in Run 3 is expected to be $2 \times 10^{34} \mathrm{~cm}^{-2} \mathrm{~s}^{-1}$ and then reach the value of $5-7$ $\times 10^{34} \mathrm{~cm}^{-2} \mathrm{~s}^{-1}$ in the HL-LHC phase. At such luminosities, the detector technologies for the innermost end-cap muon station (the Small Wheel) of the ATLAS detector [2] need to be upgraded. The Small Wheels (SW) face a huge background rate and after the luminosity upgrade, the expected rate will be as high as $15 \mathrm{kHz} / \mathrm{cm}^{2}$. The existing detector technologies of the SW are not compatible with this rate. The new technology should be able to meet the demands of good position resolution, high efficiency, fast

$\ddagger$ Copyright 2020 CERN for the benefit of the ATLAS Collaboration. CC-BY-4.0 license. 


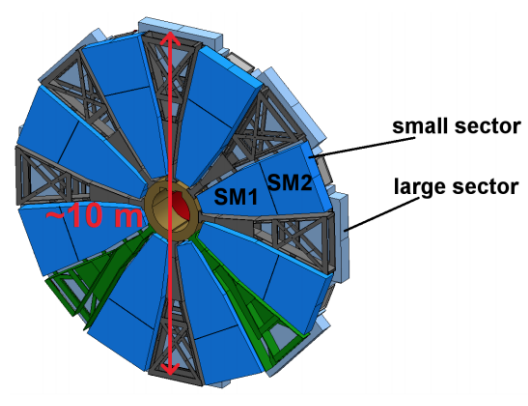

(a)

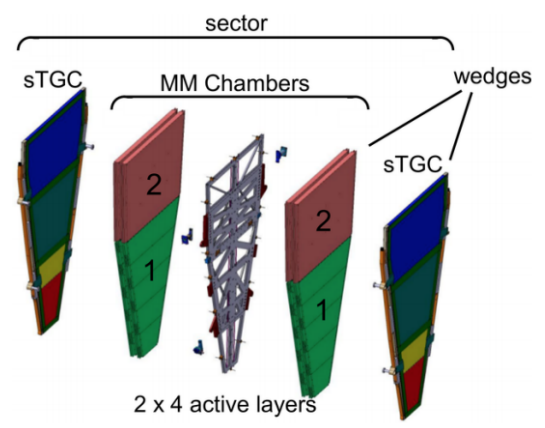

(b)

Figure 1. The structure of the NSW [3]. (a) The Wheel with the Large and Small Sectors. (b) The sTGC and the MM wedges.

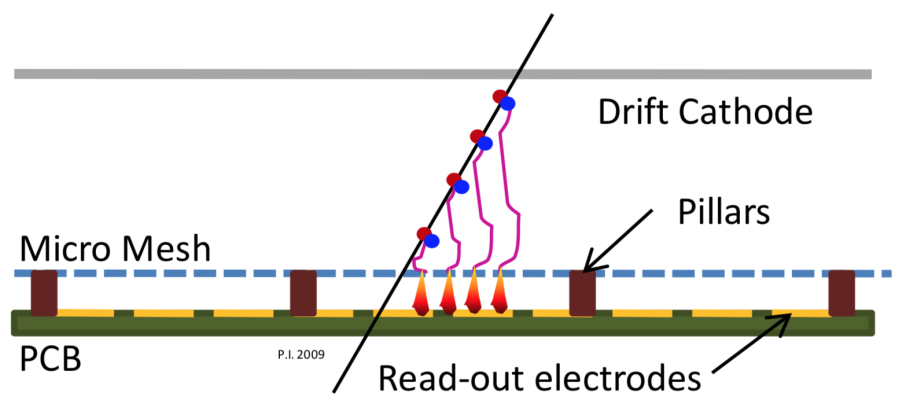

Figure 2. Schematic and working principle of a Micromegas [3].

response at the expected high background rate. The detectors for precision tracking and triggering at the New Small Wheel (NSW) [3] will be Micromegas (MM) and small-strip Thin Gas Chamber (sTGC). The detectors are also complementary to each other. Moreover, the participation of the NSW in Level-1 (L1) trigger will mitigate fake triggering at the end-cap muon stations.

Each of the two New Small Wheels comprises 8 large and 8 small sectors (Figure 1). A sector is a combination of the sTGC wedges on either side of a double Micromegas wedge. The large and the small sectors have a small overlap of the active area. A single Micromegas wedge (for both large and small) is a combination of two quadruplet modules, type-1 and type-2. Therefore, a MM double wedge (DW) is a combination of two type-1 quadruplets and two type-2 quadruplets. This allows the muon tracks to be reconstructed with $8 \mathrm{MM}$ readout layers. The total active readout area of all the Micromegas chambers for the NSWs is about $\sim 1280 \mathrm{~m}^{2}$. The structure and the construction of the Micromegas quadruplets are explained in the next sections. 


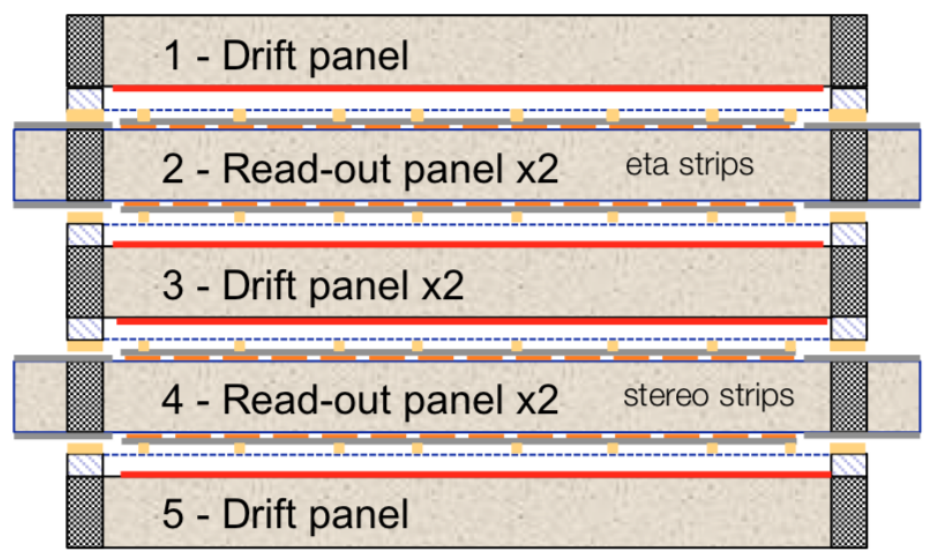

Figure 3. The schematic of a Micromegas quadruplet shows the drifts and the readout layers.

\section{The Micromegas for the NSW}

\subsection{Working principle}

Micromegas [4] belongs to the advanced generation of the gaseous detector technology, called the Micro-Pattern Gaseous Detectors (MPGD) [5]. It consists of a conversion gap or drift gap and an amplification gap. The drift gap is $5 \mathrm{~mm}$ wide and is defined by a drift cathode and a fine metallic mesh, called the micro-mesh. The micro-mesh, with the help of the dielectric pillars, is kept at a fixed distance of $128 \mu \mathrm{m}$ from the readout plane (Figure 2). The primary electrons created in the drift gap by the incident radiation (muon for instance) are driven to the amplification gap through the micro-mesh. The ratio of the drift field and amplification field is important in this process and guarantees an almost total electron-transparency of the micro-mesh. The primary electrons, due to the high electric field in the amplification gap, produce electron-avalanches. The anode is provided with resistive strips where the signal is induced. Below the resistive strips, copper strips with the same pitch allow the read-out of the signal. The resistive Micromegas concept is adopted to reduce possible sparks and allow operation of the detector in a high-radiation environment [6].

\subsection{Benefits}

Being an MPGD, Micromegas is a precise and fast detector, which makes it a suitable choice for precision tracking as well as triggering and a better replacement of the drift tubes. Position resolution of $\sim 100 \mu \mathrm{m}$ can be achieved with these chambers [7]. The readout strip pitch $(\sim 400 \mu \mathrm{m})$, is smaller than that of the drift tubes $(3 \mathrm{~cm})$, which is an advantage for track separation. Moreover, the drift time in the MM chambers is $(\sim$ $100 \mathrm{~ns})$ smaller than that of the drift tubes $(\sim 700 \mathrm{~ns})$, which makes it relatively faster. 


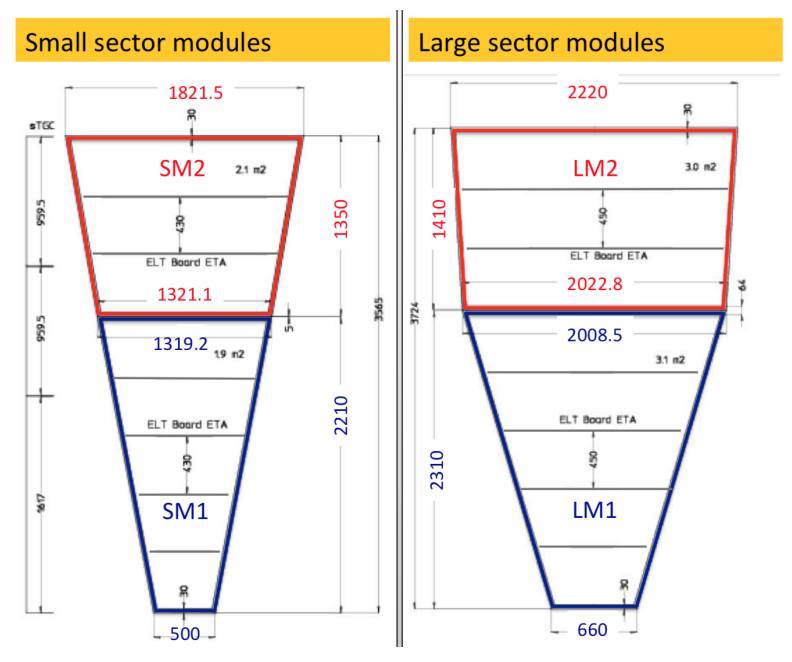

Figure 4. Dimensions of the type-1 and type-2 modules of both large, small sizes. Arrangements show how a Micromegas wedge is formed. More details can be found in [3].

\subsection{Specifications}

The micro-mesh used in the Micromegas chambers for the NSW has the wire diameter of $30 \mu \mathrm{m}$ and wire pitch of $71 \mu \mathrm{m}$. The mesh is not embedded with the dielectric pillars, but it just rests on the pillars. A quadruplet has 4 gas gaps called layers (as shown in Figure 3 ). The first two layers with strips orthogonal to the precision $\eta$ coordinate are called the $\eta$ layers, while the other 2 are the stereo layers where the readout strips are tilted by respectively $+1.5^{\circ}$ and $-1.5^{\circ}$, allowing a coarse determination of the second coordinate. The copper strips have $300 \mu \mathrm{m}$ width with a pitch of 425 or $450 \mu \mathrm{m}$ covered by an insulating layer where the resistive strips lie. A strip resistivity of $\sim 10 \mathrm{M} \Omega / \mathrm{cm}$ has been chosen in order to mitigate any possible discharge, still fulfilling the ATLAS requirement of rate capability. The primary choice of gas mixture is $\mathrm{Ar}(93 \%): \mathrm{CO}_{2}(7 \%)$, however, studies on gas mixture are going on. The choices for the drift and the amplification fields are $600 \mathrm{~V} / \mathrm{cm}, 44.5 \mathrm{kV} / \mathrm{cm}$ respectively. The mesh remains at ground potential and the resistive strips are biased at a nominal voltage of $570 \mathrm{~V}$.

\subsection{Construction}

A Micromegas quadruplet is a combination of 3 drift panels and 2 readout panels. The structure of a quadruplet is explained in Figure 3. There are two single-sided drifts on either side of one double-sided drift. The position of the readout panels are also indicated in Figure 3. There are four different sizes of quadruplets: LM1, LM2, SM1, SM2, where L, S, M stand for large, small and modules respectively. All have trapezoidal sizes as explained in Figure 4. The construction of the quadruplets are done at different sites. The LM1s are prepared in France (CEA, Saclay), LM2s are prepared in Greece (Thessaloniki) and Russia (Dubna) and CERN. The SM1s are prepared in Italy/INFNs (Pavia, Rome1, Rome3, Frascati, Lecce, Cosenza, Napoli) and the SM2s are prepared 


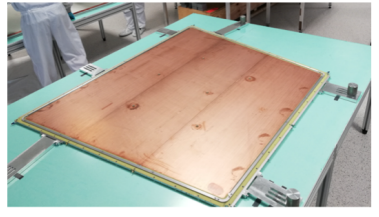

(a)

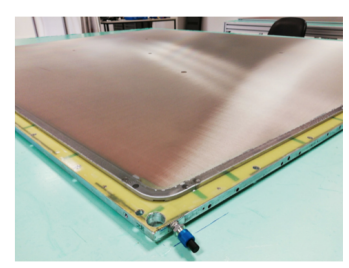

(c)

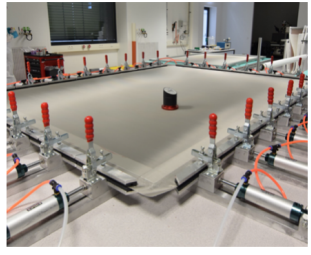

(b)

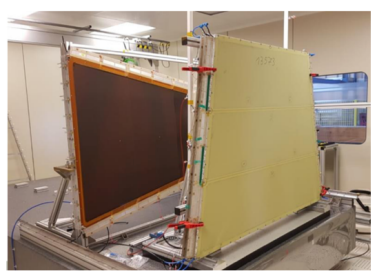

(d)

Figure 5. Example of a few construction steps of the SM2 quadruplets. (a) a bare drift panel. (b) stretching a mesh and measuring the tension. (c) a completed drift panel with micro-mesh. (d) assembling the drift and readout panel.

in Germany (Munich, Freiburg, Würzburg, Mainz).

The dimensions of the Micromegas chambers are shown in Figure 4. Producing MPGDs of such large size is extremely challenging. A very thin amplification gap $(128 \mu \mathrm{m})$ is to be maintained over $2-3 \mathrm{~m}^{2}$ area. Uniformity of the gaps, alignment, planarity, mesh-tension, cleanliness, quality of the micro-mesh and the readout etc are extremely important factors to ensure the successful performance of the chambers. All the steps involved in the entire production process are very delicate. Their detailed description is beyond the scope of this report. In general, the two major steps while preparing a quadruplet are: (a) stretching the micro-mesh, producing the drift gaps, (b) preparing and assembling the readout panel with the drift panel. Not to mention, building the panels is itself another dedicated process. Figure 5 shows the examples of a few intermediate steps of SM2 production. Cleaning the micro-mesh, measuring the uniformity of mesh tension are a few examples where extreme care is taken. For the readout panels, cleaning, gas tightness, planarity tests are performed with extreme care to qualify the chambers. Finally, after testing the quadruplets at the construction sites, they are sent to CERN.

\section{Results}

After receiving the MM-quads at CERN, first they are tested for gas tightness and highvoltage stability (with and without Gamma radiation). After that, they are integrated to form a (large or small) Micromegas double wedge. The procedures include alignment testing of the modules. After the mechanical integration is done, the double wedge is equipped with all the electronic boards. In a double wedge, there are 16 MMFE8 (Front- 


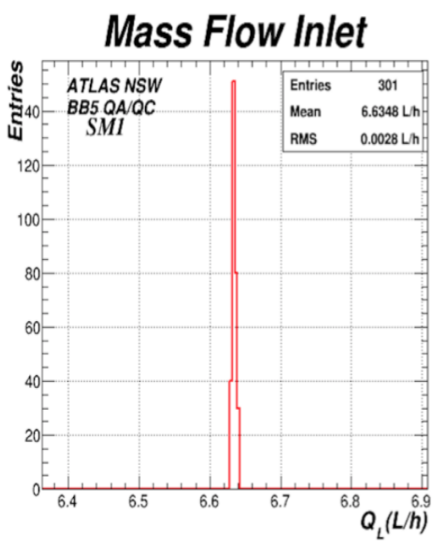

(a)

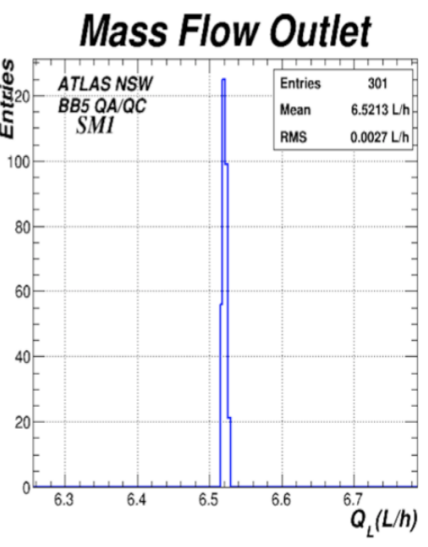

(b)

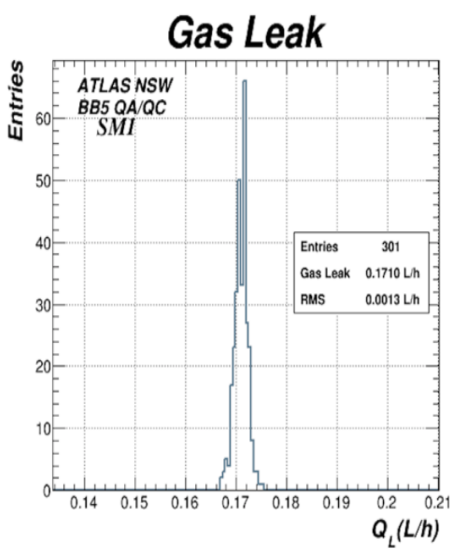

(c)

Figure 6. Gas leak test [8]. (a) Inlet flow. (b) Out-late flow. (c) Gas leak rate.

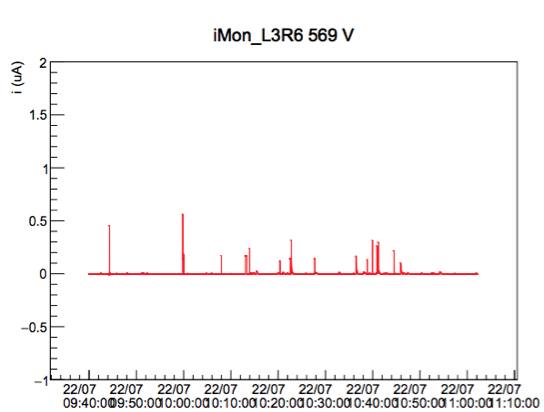

(a)

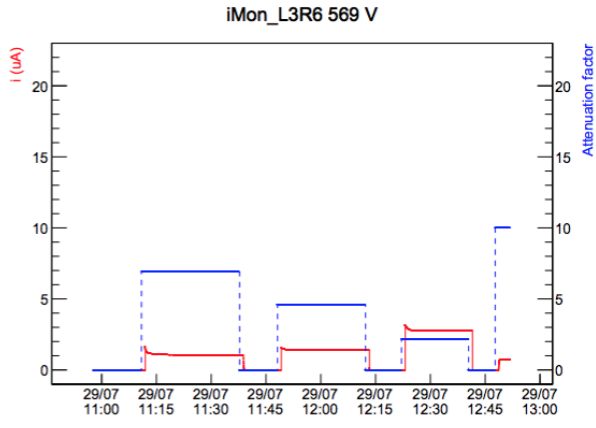

(b)

Figure 7. Examples of HV behavior of a chamber section without (a) and with (b) gamma ray irradiation. In both figures, the $\mathrm{HV}$ is kept at the nominal voltage and is shown in red the measured current. In (b) the blue line represent the gamma ray source attenuation (the higher is the level the more attenuated is the source flux). In (a) a spark rate close to the acceptance limit of 1 spark per minute is shown; in (b), the levels of currents are due to the irradiation and clearly scale with the inverse of the attenuation.

end Electronics) cards per layer which receives signal directly from the strips. Also, 2 L1DDC (Level-1 Data Driver Card for position/time information) and 2 ADDC (ART Data Driver Card, for trigger information) cards are assigned per layer. Connectivity tests and some performance tests on all these cards are also done in BB5 (the CERN site for qualifying the Micromegas DWs). This is another interesting topic which is beyond the scope of present discussion. 


\subsection{Gas leak tests}

All the quadruplets are tested for gas leak at the CERN site (BB5). It is estimated by Flow Rate Loss (FRL), where, FRL = input flow rate-output flow rate. The results are presented in Figure 6 for a chamber as an example. The test is done with an inflow rate of $6.638 \mathrm{~L} / \mathrm{h}$, at a pressure difference of 2.5 mbar. The measured leak rate at these settings is $0.171 \mathrm{~L} / \mathrm{h}$ (offset corrected), which is within the acceptance limit of ATLAS.

\subsection{HV tests}

At CERN (BB5), each quadruplet undergoes a high-voltage (HV) stability test to make sure that the HV behavior is consistent with the one seen at the construction site. The readout is segmented in many (the number depends on the type of the quadruplet) $\mathrm{HV}$-sections where the HV can be applied individually. The HV-sections should reach the nominal value of $570 \mathrm{~V}$ with no sparks or a spark rate not exceeding 6 per minute (Figure 7a). If the spark rate exceeds such a value, the section is not ramped but is kept at a lower voltage. For modules with several HV-sections kept at lower voltages, a further stability test is performed under irradiation at the Gamma ray source of the Gif ++ facility of CERN. Figure $7 \mathrm{~b}$ shows an example of the radiation test of one HVsection of a Micromegas module. The blue curve in Figure $7 \mathrm{~b}$ is the attenuation factor of the irradiation and the red curve shows the detector current.

\subsection{Test with Cosmic Muons}

Finally, when the double wedge is fully equipped with electronics, it is placed at the Cosmic Stand of BB5. A pair of two-fold scintillator coincidence is used for muon triggering. The effective trigger rate is $\sim 120 \mathrm{~Hz}$. With the cosmic rays, the readout connectivity of the electronics, the efficiency of readout layers, cluster-size, strip-multiplicity of the DW are tested. A large discrepancy, like poor efficiency of the chambers may lead to the disqualification of the MM double wedge. The first full double wedge has been tested during late August 2019. The muon tracks have been reconstructed with all eight layers. Figure 8 shows an example of such a muon track (from event display) from the first double wedge on the cosmic stand of BB5.

\section{Conclusion}

For LHC Run 3, one of the two detector technologies for the upgrade of the innermost end-cap muon stations of the ATLAS detector is Micromegas. The readout area $(\sim$ $1280 \mathrm{~m}^{2}$ ) covered by Micromegas at the New Small Wheels is unprecedented. In total around 2.1 million channels will be readout from the NSWs. From the construction of the Micromegas chambers to the qualification of the sectors for the wheel, a great deal of work is done in a systematic way, with a high level of accuracy in every step. Many challenging issues and interesting problems appeared and they have been solved. The 


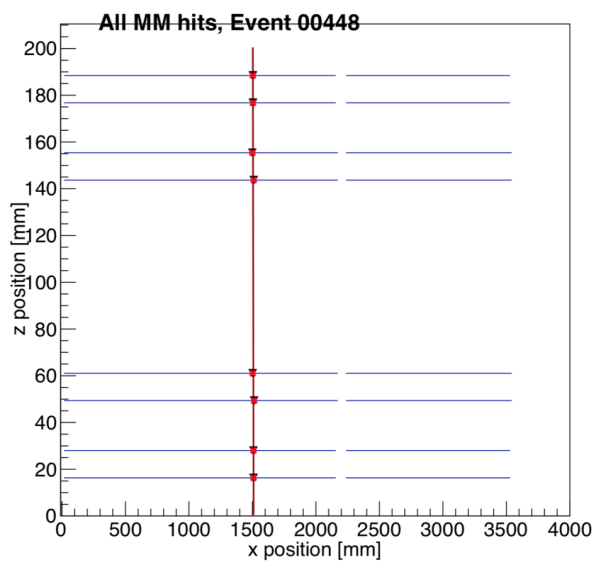

Figure 8. Example of a fully reconstructed cosmic muon event. The hits are reconstructed on all the eight layers of the first Micromegas Double-Wedge.

first Micromegas sector is already on the wheel and presently the collaboration is on its way to finishing all the sectors for the wheels.

\section{References}

[1] L.Evans and P.Bryant, LHC machine, 2008 JINST 3 S08001.

[2] ATLAS Collaboration, The ATLAS Experiment at the CERN Large Hadron Collider, JINST 3 S08003.

[3] T. Kawamoto et al, New Small Wheel Technical Design Report, CERN-LHCC-2013-006. ATLASTDR-020 (2013).

[4] Y. Giomataris et al, MICROMEGAS: a high-granularity position-sensitive gaseous detector for high particle-flux environments, NIMA 376 (1996) 29-35.

[5] M. Hoch, Trends and new developments in gaseous detectors, NIMA 535 (2004) 1-15

[6] T. Alexopoulos et al., A spark-resistant bulk-Micromegas chamber for high-rate applications, Nucl. Instrum. Meth. A 640 (2011) 110.

[7] C. Bini on behalf of the ATLAS Muon and MAMMA Collaborations, Study of the performance of the Micromegas chambers for the ATLAS muon spectrometer upgrade., JINST 9 C02032 2014.

[8] ATLAS Collaboration, ATL-MUON-PROC-2019-012. http://cds.cern.ch/record/2704673/files/ATLMUON-PROC-2019-012.pdf 